\title{
Optimization of pH Elution Conditions in Immune Complexome Analysis for Comprehensive Identification of Immune Complex Antigens
}

\author{
Nozomi Aibara, Rika Aizawa, Mikiro NaKashima, and Kaname Ohyama ${ }^{\dagger}$ \\ Unit of Medical Pharmacy, Department of Pharmacy Practice, Graduate School of Biomedical Sciences, \\ Nagasaki University, 1-7-1 Sakamoto, Nagasaki, Nagasaki 852-8588, Japan
}

\begin{abstract}
The identification of antigens incorporated into immune complexes (IC-antigens) is important for studying the pathophysiology of immunological diseases. Immune complexome analysis identifies IC-antigens by analyzing ICs collected from biological fluids by IC-capturing beads. In this study, we optimized the method to improve its comprehensiveness while maintaining selectivity for IC-antigens by comparing the number of identified peptides (model IC experiment) or proteins (human pooled serum) eluted from Protein $\mathrm{G}$ beads using different $\mathrm{pH}$ solutions ( $\mathrm{pH} 2.0$ - 11.0).
\end{abstract}

Keywords Mass spectrometry, pH elution, papain-mediated elution, immune complex antigen, immunological diseases

(Received March 31, 2020; Accepted May 28, 2020; Advance Publication Released Online by J-STAGE June 5, 2020)

\section{Introduction}

Immune complexes (ICs), formed by foreign- or self-antigens and antibodies, can damage various tissues and lead to the progression of immunological disorders, such as autoimmune diseases. ${ }^{1}$ It is therefore important to identify antigens incorporated into ICs (IC-antigens) as target molecules when developing diagnosis tools and studying the pathogenesis of a disease. Methodologies for identifying IC-antigens could differ from and be more useful than those for free antigens because ICs are real-time products of an immune response by humoral immunity. There are currently several methods for identifying IC-antigens, but few analytical methods for identifying antigens that form ICs in vivo. ${ }^{2-4}$

We have developed a method called, "immune complexome analysis", to comprehensively identify the antigens in ICs. ${ }^{5}$ In this method, ICs are isolated from biological fluids by antibodycapturing beads, such as Protein G-coated beads, tryptically digested on the beads, then analyzed by nano-liquid chromatography-tandem mass spectrometry (nano-LC-MS/MS). However, this method detects not only IC-antigens, but also large amounts of antibodies and proteins that non-specifically bind to the beads, restricting the identification of minor IC-antigens and producing false-positive (an error identification of an antigen that is not present in reality) identifications of antigens. We therefore previously attempted to selectively elute antigens from ICs captured on the beads before tryptic digestion, enhancing the selectivity and sensitivity of IC-antigen identification, by comparing papain-mediated elution and low $\mathrm{pH}$ elution with glycine/ $\mathrm{HCl}$ buffer $(\mathrm{pH} 2.5){ }^{6} \quad$ Papain is a cysteine protease that selectively cleaves the hinge region of immunoglobulin into one Fc and two identical Fab fragments. ${ }^{7,8}$ The IC-antigens and Fab fragments can be selectively eluted

† To whom correspondence should be addressed.

E-mail: k-ohyama@nagasaki-u.ac.jp from ICs bound to the beads. Low pH elution, in which solvent polarity as well as electrostatic and hydrophobic interactions between antigens and antibodies are reduced, is frequently used to separate antigens and antibodies, ${ }^{9}$ and is effective for recovering IC-antigens from ICs. We previously reported that papain-mediated elution allowed highly selective and sensitive nano-LC-MS/MS analysis of IC-antigens without interference by other proteins. ${ }^{6}$ Although low $\mathrm{pH}$ elution provides lower selectivity because antibodies also elute off the beads, it allows the identification of more IC-antigens than papain-mediated elution.

We attempted to further optimize the low $\mathrm{pH}$ elution conditions so as to improve the comprehensiveness of immune complexome analysis for identifying IC-antigens. The effect of the $\mathrm{pH}$ in the range 2.0 to 11.0 on the selective elution of IC-antigens from ICs was investigated by comparing the number of peptides derived from a model IC-antigen (complex formed by equine myoglobin and goat anti-myoglobin antibody). We also compared the number of proteins in human pooled serum eluted by buffers at the different $\mathrm{pH}$ values.

\section{Experimental}

\section{Reagents and chemicals}

Equine myoglobin and goat anti-myoglobin antibody were obtained from Sigma (St. Louis, MO, USA) and Bethyl Laboratories (Montgomery, TX, USA), respectively. Magnetic beads with immobilized Protein $G$ were purchased from Millipore (Billerica, WI, USA). Dithiothreitol, formic acid, ultrapure water, acetonitrile, acetic acid, sodium hydroxide, citric acid, and phosphate-buffered saline (PBS, $9.0 \mathrm{mmol} / \mathrm{L}$ $\mathrm{Na}_{2} \mathrm{HPO}_{4}, 2.9 \mathrm{mmol} / \mathrm{L} \mathrm{NaH} \mathrm{PO}_{4}, 137 \mathrm{mmol} / \mathrm{L} \mathrm{NaCl}, \mathrm{pH} 7.4$ ) were obtained from FUJIFILM Wako Pure Chemical Corporation (Osaka, Japan). Iodoacetamide was purchased from Tokyo Kasei Kogyo (Tokyo, Japan). Ammonium hydrogen carbonate, trifluoroacetic acid, tris(hydroxymethyl)aminomethane, disodium 
hydrogen phosphate, and hydrochloric acid were obtained from Nacalai Tesque (Kyoto, Japan). Glycine was obtained from Kanto Chemical (Tokyo, Japan). Trypsin was obtained from Promega (Madison, WI, USA).

Elution solutions with different $\mathrm{pH}$ values were prepared as follows: $\mathrm{pH} 2.0,2.5$, and 3.0 were prepared by mixing $0.1 \mathrm{M}$ glycine solution and $0.2 \mathrm{M}$ hydrochloric acid solution; $\mathrm{pH} 4.0$ and 6.0 were prepared with $0.1 \mathrm{M}$ citric acid monohydrate solution and $0.2 \mathrm{M}$ disodium hydrogen phosphate solution; pH 8.0 was prepared with $0.1 \mathrm{M}$ tris(hydroxymethyl)aminomethane solution and $0.2 \mathrm{M}$ hydrochloric acid solution; and $\mathrm{pH} 10.0$ and 11.0 were prepared with $0.1 \mathrm{M}$ glycine solution and $0.1 \mathrm{M}$ sodium hydrate solution. In this experiment, we define $\mathrm{pH} 2.0-3.0,4.0-6.0$, and $8.0-11.0$ as low range, medium range, and high range of $\mathrm{pH}$, respectively.

\section{Procedure}

Myoglobin-IC $(2.5 \mu \mathrm{M}, 10 \mu \mathrm{L})$, myoglobin $(2.5 \mu \mathrm{M}, 10 \mu \mathrm{L})$, and human pooled serum $(10 \mu \mathrm{L})$ were separately incubated with Protein G-coated magnetic beads $(20,20$, and $40 \mu \mathrm{L}$, respectively) for $30 \mathrm{~min}$ with gentle mixing. Myoglobin-IC was formed from myoglobin and anti-myoglobin at a molar ratio of 1 to 1 at room temperature for $3 \mathrm{~h}$. The liquid was removed with a pipette and the beads were washed three times with $500 \mu \mathrm{L}$ of PBS. We then added each $\mathrm{pH}$ solution ( $\mathrm{pH} 2.0$ - 11.0) and mixed them at room temperature for $10 \mathrm{~min}$. The supernatant was transferred to another tube and $10 \mathrm{mM}$ dithiothreitol ( $30 \mu \mathrm{L}$ for model IC, $50 \mu \mathrm{L}$ for serum) was added to both the supernatant eluted-fraction and the remaining beads (bead-fraction). After incubating at $56^{\circ} \mathrm{C}$ for $45 \mathrm{~min}$ for reduction, $55 \mathrm{mM}$ iodoacetamide $(30 \mu \mathrm{L}$ for model $\mathrm{IC}, 50 \mu \mathrm{L}$ for serum) was added to both fractions for alkylation. We added $10 \mu \mathrm{L}$ of trypsin $(20 \mathrm{ng} / \mu \mathrm{L}$ for myoglobin and myoglobin-IC, $200 \mathrm{ng} / \mu \mathrm{L}$ for serum) to each fraction and incubated overnight at $37^{\circ} \mathrm{C}$. At this time, $30 \mu \mathrm{L}$ of $50 \mathrm{mM}$ ammonium hydrogen carbonate solution was added to the fraction eluted by a pH 2.0 solution to adjust to neutral $\mathrm{pH}$ for tryptic digestion. We then added $10 \%$ trifluoroacetic acid to stop the digestion. Each supernatant was vacuum-reduced to approximately $30 \mu \mathrm{L}$ for model IC and $50 \mu \mathrm{L}$ for serum and the peptide mixture $(1 \mu \mathrm{L})$ was analyzed by nano-LC-MS/MS. We performed a pretreatment three times and analyzed each of the three pretreated samples in duplicate. The procedure for papain-mediated elution was performed as previously reported. ${ }^{6}$

\section{Apparatus}

Experiments were performed on a nano-LC-MS/MS system, consisting of a Shimadzu LC pump with an LC flow splitter (Dionex), an HCT PAL autosampler (CTC Analytics, Zwingen, Switzerland) and an electrospray ionization (ESI)-MS/MS instrument (LTQ XL, Thermo Fisher Scientific, Waltham, MA, USA). A nano-LC column $(\mathrm{C} 18,75 \mu \mathrm{m}$ i.d. $\times 125 \mathrm{~mm}, 3 \mu \mathrm{M}$ particle, $100 \AA$ pore size, Nikkyo Technos, Tokyo, Japan) was used for separating peptides. The peptide separation was performed with mobile phase A ( $0.1 \%$ formic acid) and mobile phase B $(0.1 \%$ formic acid in $90 \%$ acetonitrile $)$. The peptides were ion-sprayed into MS at a spray voltage of $1.5 \mathrm{kV}$, and the transfer capillary temperature was set at $200^{\circ} \mathrm{C}$. MS/MS data were extracted using a Proteome Discoverer 1.3.1.339 (Thermo Fisher Scientific). Spectra were searched against sub-databases from the public nonredundant protein database of the UniProt Knowledgebase (human, 2015.01.29 download), National Center for Biotechnology Information (equine, 2011.12.20 download; goat, 2016.5.21 download). The Tukey honestly significant difference test $(\alpha=0.05)$ was used for data comparison (a)

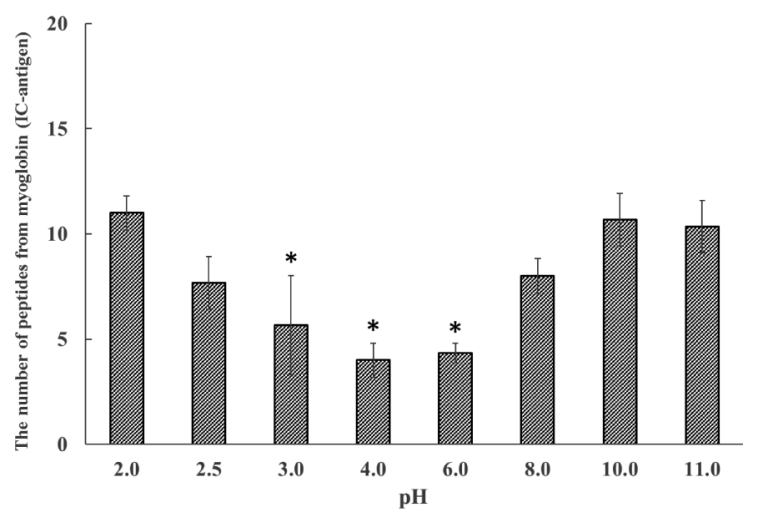

(b)

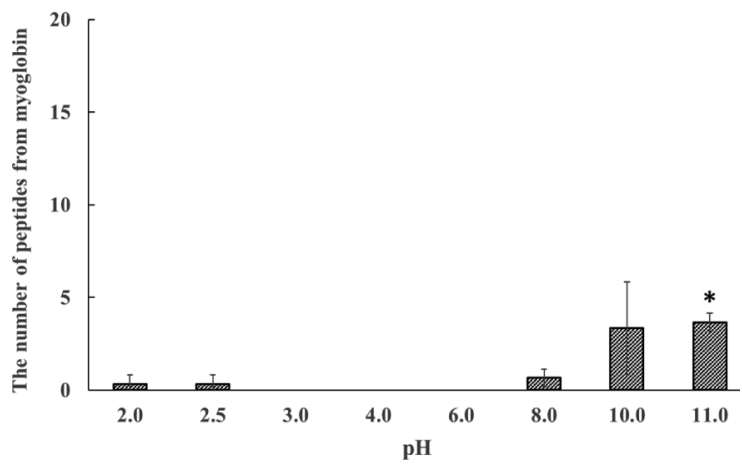

Fig. 1 (a) Number of identified peptides from myoglobin (ICantigen) in the eluted-fraction when myoglobin-IC was mixed with Protein G-coated beads. The mean values \pm SD are plotted. All statistical differences are Tukey honestly significant difference test comparisons against $\mathrm{pH} 2.0$ : $* P<0.05$. (b) Number of identified peptides from myoglobin in the eluted-fraction when only myoglobin was mixed with Protein G-coated beads. The mean values \pm SD are plotted. All statistical differences are Tukey honestly significant difference test comparisons against $\mathrm{pH} 2.0$ : $* P<0.05$.

regarding the numbers of peptide or protein between different $\mathrm{pH}$ values. Further details about this analysis were described as previously reported. ${ }^{6}$

\section{Results and Discussion}

In a previous report, ${ }^{6}$ we used three proteins (myoglobin, albumin, and alcohol dehydrogenase) as a model of IC-antigen and concluded that myoglobin was the most effective model. In the present work, we therefore evaluated the effect of different $\mathrm{pH}$ values on the comprehensiveness and selectivity of identifying IC-antigens by comparing the number of peptides from myoglobin, or the number of proteins (antigens) from serum, between the eluted-fraction and the bead-fraction. An optimized $\mathrm{pH}$ should allow the MS/MS identification of a large number of peptides from a model IC-antigen (myoglobin), and a large number of IC-antigens from pooled serum, while reducing the elution of proteins non-specifically bound to the Protein $\mathrm{G}$ beads.

\section{Model IC experiment}

We generated a model IC made by mixing myoglobin and anti-myoglobin antibodies and compared the number of peptides 
derived from myoglobin eluted by the different $\mathrm{pH}$ solutions in both fractions (eluted- and bead-fraction). We also mixed the beads with myoglobin alone as a model of non-specifically bound proteins. When myoglobin-IC was mixed with Protein G-coated beads, and then eluted with each $\mathrm{pH}$ solution, the number of myoglobin (IC-antigen) peptides in the elutedfraction using high and low range of $\mathrm{pH}$ solutions was significantly larger than the medium range of $\mathrm{pH}$ solutions (Fig. 1a). In contrast, the number of myoglobin peptides in the bead-fraction using a high or low range of $\mathrm{pH}$ solutions was smaller than the medium range of $\mathrm{pH}$ solutions (data not shown). In general, proteins are denatured at $\mathrm{pH}$ extremes and their protonation or deprotonation at ionizable groups results in repulsion. ${ }^{10}$ This means that high and low range of $\mathrm{pH}$ solutions elute myoglobin (as a IC-antigen or a non-specifically bound protein) due to denaturation, but the medium range of $\mathrm{pH}$ solutions do not elute myoglobin from the beads because of a lack of denaturation. When only myoglobin was mixed with Protein G-coated beads, very few myoglobin peptides from low range of $\mathrm{pH}$ were detected in the eluted-fractions, but more were detected at the high range of $\mathrm{pH}$ (Fig. 1b). Also, no myoglobin peptides were detected at the medium range of the $\mathrm{pH}$. On the other hand, we identified myoglobin peptides from the beadfractions at all $\mathrm{pH}$ values (data not shown). These results show that a non-specifically binding protein eluted from the beads at the high range of the $\mathrm{pH}$. The results obtained using myoglobinIC and myoglobin alone show that low range of $\mathrm{pH}$ solutions effectively elutes ICs from the Protein G-coated beads without eluting non-specifically bound proteins. One of the detailed MS/MS data of myoglobin is presented in Supporting Information (Fig. S1).

\section{Human pooled serum experiment}

We mixed $10 \mu \mathrm{L}$ of pooled serum with Protein G-coated beads, eluted with each $\mathrm{pH}$ solution, and then compared the number of IC-antigens identified in the eluted-fraction and bead-fraction for each $\mathrm{pH}$ solution. Proteins other than antibodies were considered to be antigens, and thus we had to take into account that these antigens might contain proteins that non-specifically bind to Protein G-coated beads. There tended to be a higher number of antigens in the eluted-fraction at low and high range of $\mathrm{pH}$ than at the medium range of the $\mathrm{pH}$ (Fig. 2a). Given the results of the model IC experiment, the number of proteins identified as an antigen at a high range of $\mathrm{pH}$ may be erroneous, and also contain many non-specifically bound proteins. In contrast, the eluted-fraction obtained using a low range of $\mathrm{pH}$ solution may contain far fewer non-specifically bound proteins than the corresponding fraction obtained using a high range of the $\mathrm{pH}$ solution. Details about protein names identified by each $\mathrm{pH}$ solution in the eluted-fraction are presented in Supporting Information (Table S1).

We also performed papain-mediated elution to evaluate the selectivity of $\mathrm{pH}$ elution by examining the overlap of proteins identified in human serum between papain-mediated elution and $\mathrm{pH}$ elution. We previously reported that papain-mediated elution led to the selective and sensitive identification of IC-antigens by reducing the interference from the $\mathrm{Fc}$ regions of antibodies and proteins that non-specifically bind to beads. ${ }^{6}$ However, this method has two drawbacks: (1) elution depends on the efficiency of papain digestion, which leads to false-negative identifications (an error identification of an antigen that is present in reality), and (2) the eluted fraction contains precipitates that hinder sequential analysis. On the other hand, $\mathrm{pH}$ elution more comprehensively identifies IC-antigens than papain-mediated elution. We examined the overlap ratio of IC-antigens between (a)

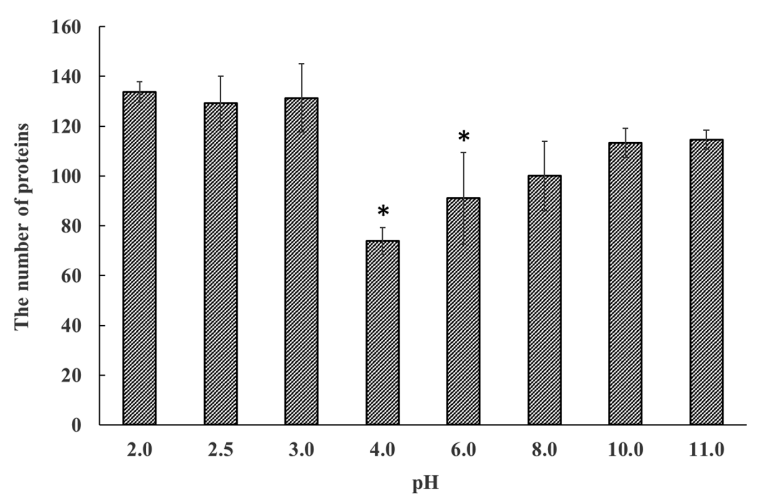

(b)

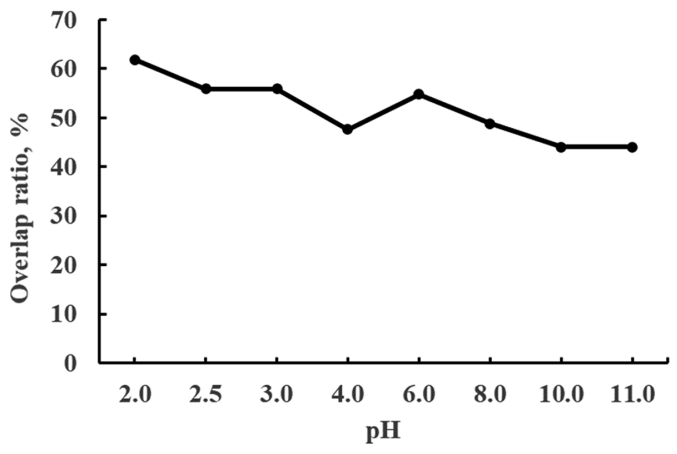

Fig. 2 (a) Number of identified proteins from serum in the elutedfraction. The mean values \pm SD are plotted. All statistical differences are Tukey honestly significant difference test comparisons against $\mathrm{pH} 2.0$ : $* P<0.05$. (b) Overlap ratio of IC-antigens between $\mathrm{pH}$ elution and papain-mediated elution.

the $\mathrm{pH}$ elution and the papain-mediated elution, and then compared these overlaps for different $\mathrm{pH}$ solutions (Fig. 2b). The largest overlap ratio was observed at $\mathrm{pH} 2.0$ and the smallest at $\mathrm{pH}$ 11.0. Papain-mediated elution supports the selective identification of IC-antigens; therefore, a large overlap ratio of $\mathrm{pH}$ elution with papain-mediated elution suggests higher selectivity to IC-antigens. In this context, $\mathrm{pH} 2.0$ elutes IC-antigens more selectively than $\mathrm{pH} 11.0$, implying that the $\mathrm{pH} 11.0$ solution elutes many non-specifically bound proteins. We therefore conclude that low range of $\mathrm{pH}$ elution (especially $\mathrm{pH} 2.0$ ) is suitable for the comprehensive detection of IC-antigens. Combining this finding with a previous study, ${ }^{6}$ the parallel use of both low $\mathrm{pH}$ elution and papain-mediated elution in immune complexome analysis may help to realize the comprehensive and selective identification of IC-antigens.

\section{Acknowledgements}

This work was supported by JSPS KAKENHI Grant Number JP19J13415 and Grant-in-Aid for JSPS Fellows. NA is also supported by Nagai Memorial Research Scholarship from the Pharmaceutical Society of Japan. This work was the result of using research equipment shared in MEXT Project for promoting public utilization of advanced research infrastructure (Program for supporting introduction of the new sharing system) Grant Number JPMXS0422500320. 


\section{Supporting Information}

MS/MS data of myoglobin (Fig. S1) and details about serum protein names identified by each $\mathrm{pH}$ solution in the elutedfraction (Table S1) are available free of charge on the Web at http://www.jsac.or.jp/analsci/.

\section{References}

1. N. Aibara and K. Ohyama, Methods Mol. Biol., 2019, 333.

2. J. H. Rho and P. D. Lampe, J. Proteome Res., 2013, 12, 2311.

3. B. Ayoglu, J. M. Schwenk, and P. Nilsson, Bioanalysis, 2016, 8,1105 .
4. J. Merl, C. A. Deeg, M. E. Swadzba, M. Ueffing, and S. M. Hauck, J. Proteome Res., 2013, 12, 5656.

5. K. Ohyama, Y. Ueki, A. Kawakami, N. Kishikawa, M. Tamai, M. Osaki, S. Kamihira, K. Nakashima, and N. Kuroda, Clin. Chem., 2011, 57, 905.

6. N. Aibara, C. Kamohara, A. K. Chauhan, N. Kishikawa, Y. Miyata, M. Nakashima, N. Kuroda, and K. Ohyama, J. Immunol. Methods, 2018, 461, 85.

7. K. G. Moorhouse, W. Nashabeh, J. Deveney, N. S. Bjork, M. G. Mulkerrin, and T. Ryskamp, J. Pharm. Biomed. Anal., 1997, 16, 593.

8. K. L. Bennett, S. V. Smith, R. J. W. Truscott, and M. M. Sheil, Anal. Biochem., 1997, 245, 17.

9. M. L. Yarmush, K. P. Antonsen, S. Sundaram, and D. M. Yarmush, Biotechnol. Prog., 1992, 8, 168.

10. H. X. Zhou and X. Pang, Chem. Rev., 2018, 118, 1691. 